\title{
Pinning-induced transition to disordered vortex phase in layered superconductors
}

\author{
A.E.Koshelev and V.M.Vinokur \\ Materials Science Division, Argonne National Laboratory, Argonne, IL 60439
}

(February 16, 2018)

\begin{abstract}
Destruction of the vortex lattice by random point pinning is considered as a mechanism of the "second peak" transition observed experimentally in weakly coupled layered high temperature superconductors. The transition field separating the topologically ordered quasilattice from the amorphous vortex configuration is strongly influenced by the layered structure and by the nonlocal nature of the vortex tilt energy due to the magnetic interlayer coupling. We found three different regimes of transition depending on the relative strength of the Josephson and magnetic couplings. The regimes can be distinguished by the dependence of the transition field on the superconductor parameters and pinning strength.
\end{abstract}

\section{INTRODUCTION.}

The influence of quenched random pinning on the crystalline order in the vortex lattice is a subject of long ${ }_{\overline{7}}$ standing interest. The early pjoneering works by Larkin and Larkin and Ovchinnikov日 introduced the picture of collective pinning in which a vortex lattice breaks up into coherently pinned domains, and these domains adjust themselves to the pinning field independently of each other. The relative displacements of vortices grow fast with the distance within these domains until they reach the characteristic scale of the pinning potential associated with the vortex core size. The fact that different Larkin domains are pinned independently suggested that any weak disorder destroys long range crystalline order. The nature of the weakly pinned vortex state was further investigated in 3 , where a concept of the low temperature vortex glass state with vortices locked near their equilibrium positions by infinite pinning barriers had been put forward. The topological nature and the consistency of the elastic description of the vortex glass has become a subject of extensive debate. It was suggested that on scales where roughness becomes of the order of the lattice constant, topological defects in the form of dislocations must appear, converting the elastic vortex glass into a liquid-like substance. This claim was however disputed in 5 where a different point of view was advocated, namely, that vortex glass induced by weak pinning preserves its elastic integrity on all scales. The conclusion oft did not account for the fact that on scales where vortex lattice roughness becomes of the order of the vortex spacing and where according tot the generation of dislocations was supposed to start, the further growth of the roughness slows down and changes from the powerlike short-scale behavion 6 to the logarithmic onet. This ultra-slow logarithmic behavior reflects the fact that as the roughness exceeds the vortex spacing, the periodic structure of the lattice becomes essential and that on the largest spatial scales vortex glass maintains quasilong-range order. This result of the seminal work by
Nattermann was later fonfirmed and dezeloped by Giamarchi and Le Doussal 19 and Korshunov 10 by using the variational replica method of Bouchaud et a 9 allowing for derivation of relations between the different energy and spatial scales within the unique approach. Structure factor $S(\mathbf{q})$ of quasilattices with quasi-long-range order (logarithmic divergence of roughness) shows power-law Bragg singularities. Owing to this property Giamarchi and Le Doussal proposed to call the glassy state resulting from weak disorder the "Bragg glass", to distinguish this state from the glassy state without any crystalline order. The possibility of dislocation-free glassy phase has also been suggested by numerical simulations of the disordered XY model by Gingras and Huse 13 .

A vortex lattice close to ideal has indeed been observed in clean $\mathrm{Bi}_{2} \mathrm{SrCa}_{2} \mathrm{Cu}_{2} \mathrm{O}_{x}$ (BiSfCO) crystals at small fields $(\lesssim 100$ Oe) by decorations 14 , neutron diffraction 15 and Lorentz microscopy 16 suggesting the existence of the topologically ordered quasilattice at low fields. At higher fields, however, the perfect lattice was never observed. This failure has been taken as the indication that elevated magnetic fields promote the action of disorder which destroys the vortex lattice. This view mas also supported by numerous transport measurement 17 which show that at high fields the resistivity components have long Arrhenius tails indicating pinning-dominated behavior. The conclusion one can draw from the above observations is that there should be a disorder-driven phase transition between the low-field quasilattice or "Bragg glass" phase and the high field topologically disordered phase. Such transition indeed has been observed in numerical simulations of Ryu et al.12 of the model of discrete lines interacting with the randomly distributed identical pinning centers. This understanding also correlates-well with the recent theoretical findings by Kierfeld et al.18 that the periodic elastic medium should remain stable with respect to weak disorder but sufficiently strong disorder can trigger the formation of topological defects in the system involved.

This putative transition can be identified with the socalled second peak field. A second peak in the mag- 
netic hysteresis loops was reported by Kopylov et al. 1 . in Tl-based HTSC compounds and by Daeumling et a for oxygen deficient $\mathrm{YBa}_{2} \mathrm{Cu}_{3} \mathrm{O}_{7-x}$ (YBCO). Further numerous investigations 2127 demonstrated that it is actually a generic feature of anisotropic and relatively clean HTSC crystals. Local magnetization measurements of Zeldov et al.28 on high quality BiSCCO single crystals reveal that the onset of the peak is very sharp, suggesting that the peak indeed may mark some phase transition. Smoothness of the peak in earlier global measurement is a consequence of spatial average due to inhomogeneous induction inside the samples. Field distribution measurements by $\mu^{+}$SR method 34 show that vortex line wandering is strongly enhanced above the onset of the peak. Investigations on BiSCCO crystals with different pxygen content29.31,32 and different Tl-based compound\$27 suggest that the peak field scales down with the decrease of the interlayer coupling. However further $\mu^{+} \mathrm{SR}$ studies by Aegerter et al. .5 show that in BiSCCO compounds with different doping the peak field correlates with the London penetration depth $\lambda_{a b}$ rather then with the interlayer couplipg and scales as $\lambda_{a b}^{-2}$. Controlled irradiation experiment 22, 33 have shown that the peak field decreases with the increase of pinning strength. These experimental facts support the interpretation of the second peak field as a field at which the vortex lattice is destroyed by pinning.

A natural question arises: why should the lattice be more ordered at smaller fields? In fact, the collective pinning theory, which for many years was the only analytical tool to study pinning phenomena, suggests exactly the opposite. By balancing the elastic and random forces, one can immediately see that random forces dominate below some typical field $B_{c p}$ and other vortices cannot prevent a given vortex to fall into the local minimum of the random potential at $B<B_{c p}$. Naively, one would expect that the vortex lattice will always be disrupted in this individual pinning regime. This fast conclusion occurs not always to be correct. In the situation when the typical distance between local minima is much smaller than the typical intervortex spacing, vortices have a wide choice of minima which gives them an extra possibility to minimize their interaction energy and restore the lattice. This situation is very typical for HTSC due to the large difference between the core size of the vortices and the intervortex spacing in the experimentally interesting field range. In order to examine vortex lattice stability with respect to weak disorder one has to compare elastic and pinning energies stored in an elementary "prism" of the vortex lattice with the base made by the elementary cell and the height equal to the characteristic elastic screening length $L_{0}$ along the field direction 30 , where $L_{0}=a_{0} / \gamma, a_{0}$ is the lattice spacing, and $\gamma$ is the anisotropy parameter. The analysis of the stability of the vortex lattice basedon the Lindemann criterion has been done recently in 36.59 .37 .38 for an anisotropic 3D superconductor. This analysis shows that the lattice is expected to be stable below some typical field and relates this field with the parameters of the superconductor and the pinning strength. Unfortunately, most of these estimates cannot be applied directly to highly anisotropic layered superconductors like BiSCCO, because in 3638 the discrete nature of the vortex lines imposed by the layered structure is not taken into account and therefore applies only to continuous elastic strings and in 39 the electromagnetic interaction between vortices was neglected.

In this paper we extend the analysis of Refs. 36, 39, 37, 38 to the case of very weakly coupled layered superconductors. Two important features distinguish layered superconductors from three dimensional superconductors. Vortex lines in layered superconductors consist of discrete segments ("pancakes"). All properties of superconductors are strongly influenced by this discrete nature of the lines. For continuous elastic strings, which are usually used to model the vortex lines, only segments of the order of the Larkin length $l_{c}$ have the possibility to explore different minima of the random potential. In layered superconductors the description in terms of elastic strings is valid only if $l_{c}$ is much larger than the separation between layers $s$. This condition is violated in the weakly coupled layered superconductors like BiSCCO. When $l_{c}$ drops below $s$, pancakes in neighboring layers acquire the possibility to explore different minima of the random potential. Vortex line wandering in such situation has been discussed in Ref. 40 in conjunction with the problem of disorder induced decoupling. Another important feature of layered compounds is that in the region where magnetic interactions between pancake vortices in different layers dominate coupling, the tilt energy of the pancake stacks becomes strongly non-local, thus modifying significantly the overall physical picture of the pinning-induced lattice destruction.

\section{PINNING-INDUCED VORTEX LINES WANDERING IN LAYERED SUPERCONDUCTORS}

Consider an isolated vortex line in a disordered layered superconductor oriented orthogonally to the layers (z-direction). Adjustment of pancake vortices to the pinning potential leads to wandering of the vortex line in the z-direction. The key parameter, which determines the amplitude of this wandering, is an elemental wandering distance $r_{\mathrm{w}} 40$ or typical distance between two pancakes in adjacent layers belonging to the same vortex line. Two distinct types of behavior emerge depending on the relation between the strength of the interlayer coupling and the pinning strength. If the distance $r_{\mathrm{w}}$ is smaller than the core size $\xi$ (weak pinning) the discreteness of vortices is not relevant, and their lateral wandering is described by the usual elastic string model. In the opposite limit, $r_{\mathrm{w}}>\xi$, the discreteness plays an essential role. Now pancakes in the neighboring layers have the possibility to stretch their relative displacements to distances much larger than the typical spacing between the minima of the 
random potential, to explore a whole lot of local minima within the area of $r_{\mathrm{w}}^{2}$ and thus choose the best of all of them.

The distance $r_{\mathrm{w}}$ is determined by the balance between the elastic tilt energy and the random energy 40 . The tilt energy of the vortex line

$$
\mathcal{E}_{\text {tilt }}\left[\mathbf{u}_{n}\right]=\mathcal{E}_{\mathrm{J}}\left[\mathbf{u}_{n}\right]+\mathcal{E}_{\mathrm{M}}\left[\mathbf{u}_{n}\right]
$$

consists of the Josephson,

$$
\mathcal{E}_{\mathrm{J}}\left[\mathbf{u}_{n}\right]=\sum_{n} \frac{\pi E_{\mathrm{J}}}{2}\left(\mathbf{u}_{n}-\mathbf{u}_{n-1}\right)^{2} \ln \frac{r_{\mathrm{J}}}{\left|\mathbf{u}_{n}-\mathbf{u}_{n-1}\right|}
$$

and magnetic,

$$
\mathcal{E}_{\mathrm{M}}\left[\mathbf{u}_{n}\right]=\frac{E_{\mathrm{M}}}{2 s} \int\left(d k_{z}\right) \ln \left(1+\frac{r_{\mathrm{cut}}^{2} Q_{z}^{2}}{1+r_{\mathrm{w}}^{2} Q_{z}^{2}}\right)\left|\mathbf{u}\left(k_{z}\right)\right|^{2}
$$

contributions. Here $\mathbf{u}_{n}$ is the pancake displacement in the $n$-th layer and $\mathbf{u}\left(k_{z}\right)$ is the corresponding Fourier transform, $\mathbf{u}\left(k_{z}\right)=s \sum_{n} \exp \left(-i k_{z} z_{n}\right) \mathbf{u}_{n} . \quad E_{\mathrm{J}}=$ $\varepsilon_{0} /\left(\pi \gamma^{2} s\right)$ and $E_{\mathrm{M}}=s \varepsilon_{0} / 2 \lambda_{a b}^{2}$ are the Josephson and magnetic energies per unit area, $\varepsilon_{0}=\Phi_{0}^{2} /\left(4 \pi \lambda_{a b}\right)^{2}, r_{\mathrm{J}}=$ $\gamma s$ is the Josephson length, $\gamma=\lambda_{c} / \lambda_{a b}$ is the anisotropy ratio of the London penetration depths $\lambda_{a b}$ and $\lambda_{c}, s$ is the interlayer spacing, and $Q_{z}^{2}=\frac{2}{s^{2}}\left(1-\cos k_{z} s\right)$. The expression under the logarithm in the magnetic coupling tilt energy describes the crossovers between different regimes depending on the relations between different length scales of the problem $\left(\lambda_{a b}, \mathrm{~s}\right.$, lattice spacing $a$, and $\left.r_{w}\right) 42$. The cut off length $r_{\text {cut }}$ can be estimated from $r_{\text {cut }}^{-2} \approx \lambda_{a b}^{-2}+21.3 B / \Phi_{0}$.

Consider a randomly misaligned vortex line with a typical relative displacement of adjacent pancakes from one stack $r \sim\left|\mathbf{u}_{n}-\mathbf{u}_{n-1}\right|$. The local energy change $\epsilon(r)$ per pancake caused by this misalignment includes the loss in the coupling energy $\epsilon_{\text {coup }}(r)$ and the gain in the random energy $\epsilon_{\text {ran }}(r), \epsilon(r)=\epsilon_{\text {coup }}(r)+\epsilon_{\text {ran }}(r)$. The coupling term is determined by the tilt energy (1) at large wave vectors $k_{z} \sim \pi / s$ and, as we mentioned above, can be split into the Josephson and magnetic contributions:

$$
\epsilon_{\mathrm{coup}}(r)=\frac{\pi}{2} E_{\mathrm{J}} r^{2} \ln \frac{r_{\mathrm{J}}}{r}+E_{\mathrm{M}} r^{2} \ln \frac{r_{\mathrm{cut}}}{r} .
$$

Note the different physical origin of these terms: while the loss in Josephson energy is related to the interaction of a given pancake with pancakes in neighboring layers, the magnetic energy is determined by the averaged interaction with pancakes from a large number $\left(r_{\text {cut }} / s\right)$ of remote layers. The term $\epsilon_{\mathrm{ran}}(r)$ describes the gain in the pinning energy upon adjusting the position of the given pancake to a best minimum of the random potential within the area $\pi r^{2}$. We consider $r \gg \xi$ (weak coupling) so that pancakes have the possibility to choose among a large number of minima of the random potential. In such a situation, the function $\epsilon_{\mathrm{ran}}(r)$ is determined by the distribution of the pinning energies, which for pinning due to point defects is natural to expect to have a Gaussian form. Accordingly, the concentration of minima with energies between $\epsilon$ and $\epsilon+d \epsilon$ is $P(\epsilon) d \epsilon$ with

$$
P(\epsilon)=\frac{\exp \left(-\frac{\epsilon^{2}}{U_{p}^{2}}\right)}{r_{\mathrm{p}}^{2} U_{\mathrm{p}}},
$$

where $U_{\mathrm{p}}$ is the pinning energy and $r_{\mathrm{p}}$ is the typical pinning size. Numerical investigation of random potential for $\delta T_{c}$-pinning give $r_{\mathrm{p}} \approx 6.3 \xi$, where $\xi$ is the coherence length. The function $\epsilon_{\mathrm{ran}}(r)$ is determined by the condition that the number of centers with pinning energy less than $\epsilon_{\text {ran }}$ in an area $\sim r^{2}$ is of the order of one, i.e., $\pi r^{2} \int^{\epsilon} P(\varepsilon) d \varepsilon \sim 1$. For large displacements, $r \gg r_{\mathrm{p}}$, this condition gives

$$
\epsilon_{\mathrm{ran}}=-U_{p} \ln ^{1 / 2}\left(\frac{r^{2}}{2 \sqrt{\pi} r_{p}^{2}}\right) .
$$

The optimization of $\epsilon(r)$ with respect to $r$ gives the following equation for $r_{\mathrm{w}}$ :

$$
r_{\mathrm{w}}^{2}=\frac{U_{p}}{\left(\pi E_{\mathrm{J}} \ln \frac{r_{\mathrm{J}}}{r_{\mathrm{w}}}+2 E_{\mathrm{M}} \ln \frac{r_{\mathrm{cut}}}{r_{\mathrm{w}}}\right) \ln ^{1 / 2}\left(\frac{r_{\mathrm{w}}^{2}}{2 \sqrt{\pi} r_{p}^{2}}\right)},
$$

which is valid if $r_{\mathrm{w}} \gg \xi$. In the limit where the interlayer coupling is not too weak, $\gamma<\lambda_{a b} / s$, the distance $r_{\mathrm{w}}$ is mainly determined by the Josephson coupling, and an approximate solution of Eq. (7) is given by

$$
r_{\mathrm{wJ}}^{2} \approx \frac{U_{p}}{\pi E_{\mathrm{J}} \ln \frac{s \varepsilon_{0}}{U_{p}} \ln ^{1 / 2}\left(\frac{U_{p}}{E_{\mathrm{J}} r_{p}^{2}}\right)}
$$

In the opposite limit of very weak coupling, $\gamma>\lambda_{a b} / s$, the wandering length is determined by the magnetic coupling,

$$
r_{\mathrm{wM}}^{2} \approx \frac{U_{p}}{E_{\mathrm{M}} \ln \frac{E_{\mathrm{M}} r_{\mathrm{cut}}^{2}}{U_{p}} \ln ^{1 / 2}\left(\frac{U_{p}}{E_{\mathrm{M}} r_{p}^{2}}\right)} .
$$

In the latter case $r_{\mathrm{w}}$ is mainly controlled by the London penetration depth $\lambda_{a b}$ and does not depend upon the anisotropy ratio at all.

Point pinning induces lateral random wandering of the vortex line as it traverses a sample in the $\mathrm{z}$-direction so that the line displacements grow as $\mathbf{u}_{n}=\mathbf{u}\left(z_{n}\right)$, which is commonly characterized by the mean squared displacement or roughness, $w\left(z_{n}\right), w\left(z_{n}\right)=\sqrt{\left\langle\left(\mathbf{u}_{n}-\mathbf{u}_{0}\right)^{2}\right\rangle}$. The shape of the function $w\left(z_{n}\right)$ is determined by the nature of interlayer coupling.

In the case of dominating magnetic coupling the energy of the mismatched pancake stack (3) is strongly nonlocal, i.e., the energy cost of pancake displacement in a given layer is not simply determined by the pancake positions in two adjacent layers but it is determined by the whole 
line configurations at distances $r_{\text {cut }}=\min \left(\lambda_{a b}, a\right)$ from the given layer. A rough estimate for the displacement field at small distances $z \ll r_{\text {cut }}$ can be obtained if we neglect weak logarithmic $k_{z}$-dependence of the tilt energy (3) which allows us to describe the collective interaction with a large number of pancakes in terms of an effective "cage potential"

$$
\mathcal{E}_{\mathrm{M}}^{\text {(cage) }} \approx E_{\mathrm{M}} \ln \left(\frac{r_{\mathrm{cut}}}{r_{\mathrm{wM}}}\right) \sum_{n} \mathbf{u}_{n}^{2} .
$$

In the "cage" approximation the correlations between the layers are absent and the displacement field $w(z)$ does not increase at all

$$
w(z) \approx r_{\mathrm{wM}}, \text { at } z<r_{\mathrm{cut}} .
$$

At small distances a weak dispersion of the tilt energy is to be taken into account perturbatively and gives rise to a weak increase of $w(z)$

$$
w(z) \approx r_{\mathrm{wM}}\left[1-\frac{s}{4 z \ln \frac{r_{\mathrm{cut}}}{r_{\mathrm{wM}}}}\right] .
$$

Only at very large distances $z \gg r_{\text {cut }}$ the nonlocality becomes irrelevant and displacements start to grow again.

The Josephson interlayer coupling provides an additional contribution to the tilt energy of a misaligned pancake stack (2) which is determined by the interactions between neighboring pancakes in adjacent layers. In the case $\gamma<\lambda_{a b} / s$ the Josephson energy dominates at small distances. Due to the locality of interactions the pinning-induced relative displacement $w\left(z_{n}\right)$ grows with $z_{n}$ similar to the elastic string:

$$
w(z)=r_{\mathrm{wJ}}(z / s)^{\zeta}
$$

The rate of growth is determined by the universal wandering exponent $\zeta$. Elaborate numerical analysis 43 gives $\zeta \approx 5 / 8$, which is larger than the naively expected "random walk" exponent $1 / 2$. It is also slightly larger that the value $3 / 5$ suggested by the renormalization group analyses 44 and by a simple scaling reasoning 6 (see also review 45 ). Relative displacements for the layered superconductor given by Eq. (13) differ from displacement for weakly pinned vortex line in 3D superconductors $w(z)=\xi\left(z / l_{c}\right)^{\zeta}$ by the elemental lengths: $r_{\mathrm{wM}}$ replaces the core size $\xi$ and the interlayer spacing $s$ comes instead of the Larkin length $l_{c}$. The 3D expression for $w(z)$, which has been used in Refs. 36 38, is valid only if $l_{c}>s$. The last condition is strongly violated in highly anisotropic layered high- $\mathrm{T}_{c}$ materials like BiSSCO and in these materials one has to use Eqs. (11,12) or Eq. (13) depending on relative strength of the Josephson and magnetic couplings.

Note that even in the case of relatively strong Josephson coupling $\left(\gamma<\lambda_{a b} / s\right)$ the growth of $w(z)$ at large distances is restricted by magnetic interactions. Comparison of the Josephson (2) and magnetic (3) tilt energies shows that above the typical length $L_{\mathrm{J}-\mathrm{M}} \approx \pi \sqrt{\frac{2}{\ln (\gamma)}} \frac{\lambda_{a b}}{\gamma}$ the magnetic coupling takes over again and the displacement field crosses over from dependence (13) to the almost $z$ independent behavior

$$
w\left(L_{\mathrm{J}-\mathrm{M}}\right) \approx r_{\mathrm{wJ}}\left(\frac{\lambda_{a b}}{\gamma s}\right)^{\zeta} .
$$

Different regimes of line wandering are illustrated in Fig. 1.

\section{DESTRUCTION OF THE VORTEX CRYSTAL BY DISORDER}

\section{A. Interaction length scale $l_{3 D}$}

Lateral displacements of an individual vortex line would grow infinitely with $z$, however the presence of other vortices restricts its wandering. A given line starts to feel its neighbors at scales exceeding some typical length $l_{3 D}$, which marks the crossover between the single vortex and three dimensional (bundle) regimes. To estimate this length one has to balance the typical tilt energy at the wave vector $\pi / l_{3 D}, C_{44}\left(\pi / l_{3 D}\right)^{2}$ and the typical shear energy $4 \pi C_{66} B / \Phi_{0}$. This gives the following estimate

$$
l_{3 D} \approx \sqrt{\frac{C_{44} \Phi_{0}}{C_{66} B}}
$$

The individual line description applies as long as $l_{3 D}$ is larger than the separation between the layers. The shear and tilt moduli for layered supercondyctor in the field range $\Phi / \lambda_{a b}^{2}<B \ll H_{c 2}$ are given by 46,42

$$
\begin{aligned}
C_{66} & =\frac{B \Phi_{0}}{\left(8 \pi \lambda_{a b}\right)^{2}} ; \\
C_{44}(\mathbf{k}) & =\frac{B^{2} / 4 \pi}{1+\lambda_{c}^{2} k_{\|}^{2}+\lambda_{a b}^{2} Q_{z}^{2}}+\frac{B \Phi_{0}}{2\left(4 \pi \lambda_{c}\right)^{2}} \ln \frac{k_{\max }^{2}}{K_{0}^{2}+\left(Q_{z} / \gamma\right)^{2}} \\
& +\frac{B \Phi_{0}}{2(4 \pi)^{2} \lambda_{a b}^{4} Q_{z}^{2}} \ln \left(1+\frac{r_{\mathrm{cut}}^{2} Q_{z}^{2}}{1+r_{\mathrm{w}}^{2} Q_{z}^{2}}\right)
\end{aligned}
$$

with $K_{0}=\sqrt{4 \pi B / \Phi_{0}}$ being the average radius of the Brillouin zone. Three terms in the tilt modulus correspond to the nonlocal collective, Josephson single vortex and magnetic contributions respectively. In our case the cutoff wave vector $k_{\max }$ in the second term of $C_{44}$ can be estimated as $k_{\max } \approx \pi / r_{\mathrm{w}}$. The nonlocal tilt modulus in Eq. (15) should be taken at $k_{z}=\pi / l_{3 D}$ and $k_{\|}=K_{0}$.

Two field regimes exist depending upon the strength of the interlayer coupling. For small Josephson coupling $\gamma>\lambda_{a b} / s$ the length $l_{3 D}$ is determined by the magnetic coupling in the whole field range and decays exponentially at $B>\Phi / \lambda_{a b}^{2}$ 


$$
l_{3 D} \approx a \exp \left(-\frac{B\left(\pi \lambda_{a b}\right)^{2}}{4 \Phi_{0}}\right) .
$$

At field

$$
B_{\mathrm{M} c r} \approx \frac{4 \Phi_{0}}{\left(\lambda_{a b} \pi\right)^{2}} \ln \frac{\lambda_{a b}}{r_{\mathrm{w}}}
$$

the length $l_{3 D}$ matches $r_{\mathrm{w}}$, indicating the crossover to the quasi-2D regime.

In the case of not too weak Josephson coupling $\gamma<$ $\lambda_{a b} / s$, two relevant length scales govern the behavior of a single vortex line: interlayer spacing $s$ and the crossover length $L_{\mathrm{J}-\mathrm{M}}$ introduced in the previous Section. One can distinguish three field regimes corresponding to different relations between $l_{3 D}$ and these lengths. At fields smaller than the typical field $B_{\mathrm{J}-\mathrm{M}}=\frac{2 \Phi_{0}}{\left(\lambda_{a b} \pi\right)^{2}} \ln \left(\frac{0.1 \gamma^{2}}{\ln \left(\lambda_{a b} / r_{\mathrm{w}}\right)}\right)$ the interaction crossover takes place at length scales larger than $L_{\mathrm{J}-\mathrm{M}}$ where magnetic coupling still dominates and the length $l_{3 D}$ is again determined by Eq. (17). At higher fields the Josephson coupling dominates and the length $l_{3 D}$ is given by

$$
l_{3 D} \approx 2 \frac{a}{\gamma} \sqrt{\ln \frac{a}{r_{\mathrm{w}}}} .
$$

The crossover to the quasi-2D regime takes place at the field $B=B_{\text {Jcr }}$ t46,

$$
B_{\mathrm{J} c r}<\frac{4 \Phi_{0}}{(\gamma s)^{2}} \ln \frac{\gamma s}{r_{\mathrm{w}}}
$$

The schematic field-anisotropy phase diagram shown in Fig. 2 summarizes the above description of the different regimes.

The field at which random pinning destroys the quasilattice or the "Bragg glass" phase pestimated from the Lindemann-like criterion. 36.39,37.38 The destruction of the lattice is expected when the displacement $w(z)$ at $z \approx l_{3 D}$ reaches some fraction of the lattice spacing

$$
w\left(l_{3 D}\right)=c_{L} a
$$

Several regimes exist depending upon the strength of coupling and the strength of pinning.

\section{B. Dominating magnetic coupling}

In the regime where magnetic coupling dominates $(\gamma>$ $\left.\lambda_{a b} / s\right)$ the line roughness $w(z)$ almost does not depend on $z$ and the criterion (21) can be written simply as

$$
r_{\mathrm{wM}}=c_{L} a,
$$

which leads to the estimate of the field at which the "Bragg glass" is destroyed:

$$
B_{x}=C_{M} \frac{\Phi_{0}}{\lambda_{a b}^{2}} \frac{T_{m}^{2 D}}{U_{p}}
$$

Here $T_{m}^{2 D} \approx s \varepsilon_{0} / 70$ is the melting temperature for a single pin-free $2 \mathrm{D}$ layer and $C_{M}=35 c_{L}^{2} \sqrt{\ln \left(\frac{2 \lambda_{a b}^{2} U_{p}}{s \varepsilon_{0} r_{p}^{2}}\right)} \approx$ $1 \div 2$ is a numerical constant weakly depending on the parameters. The melting temperature gives a natural scale for the pinning strength. It is important to note that the relative displacement of pancakes in neighboring layers becomes comparable with the lattice spacing about the same field $B_{x}$. This means that misalignment transition and disorder-induced destruction of the vortex lattice merge and that above $B_{x}$ one is going to find the state with both completely misaligned and disordered configuration of pancakes. This is very similar to merging of the lattice disordering and the misalignment transitions induced by the magnetic coupling first pointed out by Blatter et al.47 in the context of the melting transition.

A very unusual feature of the transition imposed by dominating magnetic coupling is that the transition field (23) is almost insensitive to the shear stiffness of the lattice. The reason is that due to the strong nonlocality of magnetic interactions the wanderings of the given line are self-confined, i.e. the mean squared displacements at relevant length scales are mainly determined by the interactions of the pancakes belonging to the same string, and interaction with other strings very weakly influences these displacements.

The disorder-induced destruction of the vortex lattice can occur only if $B_{x}$ lies below the dimensional crossover field $B_{\mathrm{Mcr}}$ (18). This gives the following condition for the pinning strength

$$
U_{p} \gtrsim 0.5 T_{m}^{2 D}
$$

which is simply a condition that pinning is strong enough to destroy the 2D lattice in a single layer. For weaker pinning the "Bragg glass" remains stable in the quasi-2D regime up to fields close to $H_{c 2}$. On the other hand, if $U_{p}$ is substantially larger than $T_{m}^{2 D}$ then the field $B_{x}$ falls into the region of exponentially weak interacting vortices. In this case the intermediate quasilattice state collapses and the vortex lattice is in a disordered state throughout the whole field range. One can conclude therefore that the intermediate quasilattice state at fields $\sim \Phi_{0} / \lambda_{a b}^{2}$ in magnetically coupled superconductors exists only within the limited range of the pinning strength where $U_{p} \sim$ $T_{m}^{2 D}$.

\section{Strong Josephson coupling}

Now we turn to the case $\gamma<\lambda_{a b} / s$. Due to the existence of the two relevant length scales controlling wandering of a single line, $s$ and $L_{\mathrm{J}-\mathrm{M}}$, the behavior is very rich. Depending on the pinning strength the destruction of the "Bragg glass" phase may take place either in the "3D Josephson-" or in the "3D Magnetic" regimes (see 
Fig. 2) or it may not happen at all. When the transition falls into the "3D Josephson" region we obtain from Eqs.(21, 13, 19) the following estimate for the transition field

$$
B_{x}=\frac{\Phi_{0}}{(\gamma s)^{2}}\left(\frac{c_{w} \gamma s}{r_{\mathrm{w}}}\right)^{2 \beta}
$$

with $\beta=\frac{1}{1-\zeta} \approx 8 / 3$ and $c_{w}=c_{L}\left(4 \ln \frac{a}{r_{\mathrm{w}}}\right)^{-\zeta / 2}=0.08 \div$ 0.1. Using Eq.(8) $B_{x}$ can also be connected with the pinning potential $U_{p}$

$$
B_{x}=\frac{\Phi_{0}}{(\gamma s)^{2}}\left(\frac{C_{J} T_{m}^{2 D}}{U_{p}}\right)^{\beta}
$$

with $C_{J}=15 \ln \frac{s \varepsilon_{0}}{U_{p}}\left(\ln \frac{\Phi_{0} E_{\mathrm{J}}}{B U_{p}}\right)^{-\zeta} \sqrt{\ln \left(\frac{U_{p}}{E_{\mathrm{J}} r_{p}^{2}}\right)} c_{L}^{2} \approx 0.2 \div$ 0.35. It is interesting to note that in this regime $B_{x} \propto 1 / \gamma^{2}$ in agreement with experimental trends29,31,32. Eq.(26) is valid provided $B_{x}$ falls into the interval $B_{\mathrm{J}-\mathrm{M}}<B_{x}<B_{\mathrm{Jcr}}$. This gives the following conditions for the elemental wandering distance

$$
0.05 \gamma s \lesssim r_{\mathrm{w}} \lesssim 0.05 \gamma s\left(\frac{\pi \lambda_{a b}}{\gamma s}\right)^{1-\zeta}
$$

and for the pinning strength

$$
0.5 T_{m}^{2 D} \lesssim U_{p} \lesssim 0.5 T_{m}^{2 D}\left(\frac{\pi \lambda_{a b}}{\gamma s}\right)^{2(1-\zeta)}
$$

For weaker pinning (smaller $r_{\mathrm{w}}$ ) the "Bragg Glass" is not destroyed by pinning. For stronger pinning (larger $r_{\mathrm{w}}$ ) the transition falls into the "3D Magnetic" regime. In this regime the criterion for lattice destruction can be written as.

$$
w\left(L_{\mathrm{J}-\mathrm{M}}\right)=c_{L} a
$$

and leads to the following estimate for the transition field

$$
B_{x}=C_{\mathrm{JM}} \frac{\Phi_{0}}{\lambda_{a b}^{2}}\left(\frac{\lambda_{a b}}{\gamma s}\right)^{2(1-\zeta)} \frac{T_{m}^{2 D}}{U_{p}}
$$

with $C_{\mathrm{JM}}=70 c_{L}^{2} \ln \frac{s \varepsilon_{0}}{U_{p}} \sqrt{\ln \left(\frac{U_{p}}{E_{\mathrm{J}} r_{p}^{2}}\right)} \approx 10-15$.

The "elastic string" regime of the transition considered in Refs. 36 38 exists at least for some pinning strength only if the crossover from the pancake pinning regime to the string pinning regime of an individual vortex line occurs at $U_{p} \gtrsim T_{m}^{2 D}$. This gives the relation $E_{\mathrm{J}} \xi^{2}>T_{m}^{2 D}$ which can be rewritten as the condition for the anisotropy $\gamma s \lesssim 20 \xi$

Different regimes of quasilattice destruction represented by Eqs. $23,26,30$ ) are summarized in the anisotropy-pinning phase diagram (Fig. 3).

\section{DISCUSSION}

Taking typical parameters for optimally doped BiSSCO, $\lambda_{a b} \approx 2400 \AA 35, \gamma=200-300, s=15 \AA$, and $U_{p}=10-15 \mathrm{~K}$ we can conclude that BiSSCO is located in region (3) of the phase diagram of Fig. 2 (region of dominating magnetic coupling) but not too far from the boundary with region (2) so that the Josephson coupling is probably not completely negligible. Estimate for the transition field in region (3) is given by Eq. (23). Substituting the parameters, we obtain $B_{x}=200-300 \mathrm{G}$, in reasonable agreement with experiments 35.29.

The above approach can specify a position for the transition line from the quasilattice to disordered solid, but can neither prove the existence of such a transition, nor describe the nature of the resulting high field entangled solid phase. The first question requiring understanding the entangled solid state is the very result that enabled us to observe this transition: what is the mechanism of the sharp increase of the persistent current measured in the magnetization experiment? Indeed, one can think that since at the moment of transition pinning in the quasilattice is dominated by the single vortex pinning regime, the critical current can only drop with the increase of the magnetic field. However as we have already mentioned in the Introduction this "classical" collective pinning approach does not account for the possibility that pancakes can explore the deep bound states. A maximum possible jump of the critical current at the transition is expected for the case of dominated magnetic coupling. In this case vortex lines are destroyed at the transition point and it is reasonable to assume that above the transition each pancake has the possibility to choose the optimum location within the area $(\pi / 4) a_{0}^{2}$. If we assume that the distribution of the energy minima $E_{m}$ for pancakes obeys Gaussian statistics $P\left(E_{m}\right) \propto \exp \left(-E_{m}^{2} / U_{p}^{2}\right)$, the typical pinning energy that a pancake can find on the available area just above the transition is estimated as

$$
E_{p i n}^{>} \simeq U_{p} \sqrt{\ln \frac{a_{0}^{2}}{\xi^{2}}}
$$

On the other hand, just below the transition point pancakes has the possibility to look for the best pinning center only within the area $u^{2}$, where $u$ is the mean squared deviation of the vortex lines from its average position, which at the transition point is given by the Lindemann relation $u=c_{L} a$. Therefore a typical pinning energy below the transition can be estimated as

$$
E_{p i n}^{<} \simeq U_{p} \sqrt{\ln \frac{c_{L}^{2} a_{0}^{2}}{\xi^{2}}}
$$

Since the critical current in a single pancake pinning regime is about $J_{c} \simeq E_{\text {pin }} / \xi$, we see that one can expect a substantial growth of the critical current at the transition. For typical BiSCCO parameters the critical 
current can jump by a factor of 2 . However the experimental jump of the persistent magnetization current is only indirectly related to the enlargement of the critical current, because in real experiment the persistent current is strongly reduced by thermal creep. The jump most probably reflects the enhancement of the relaxation barrier above the transition as was indeed observed in recent elaborated creep measurements of Konczykowski et al.49. Quantitative description of this enhancement is a challenging problem due to the collective nature of relaxation both below and above the transition.

Another important question is whether the entangled solid is a glass or the glassy state is destroyed by a massive proliferation of dislocations. A very naive expectation is that dislocations convert the vortex solid into a liquid, infinite barriers for vortex motion disappear and collective creep over divergent barriers transforms into a plastic creep governed by the motion of dislocation over the finite Peierls barriers. More attentive (although still naive) analysis shows however, that being linear elastic objects, dislocations may be pinned in turn recovering the glassy response of the entangled solid. Indeed, recent relaxation measurements on YBCO crystal 48 indicate that creep activation barriers show the linear current dependence in a wide range of currents and only at very small currents some upturn in current dependence of energy is observed, suggesting the appearance of diverging barriers. On the other hand, it is not quite clear whether this naive dislocation-based approach applies to highly anisotropic superconductors. One can notice that neglecting the Josephson coupling one arrives at creep governed by two-dimensional pancake diffusion in the entangled phase. Since the diverging barriers do not appear for 2D particle diffusion, the presence of electromagnetic coupling only cannot provide glassiness. One can notice however that switching on even weak Josephson coupling recovers glassy response, giving rise to a phase glass (or dislocation glass, or plastic glass) phase above the entanglement transition. The glassy properties of the entangled solid will be discussed in detail elsewhere. Here we point out only that the recent experimental ghservation on BiSCCO crystals by Konczykowski et al 49 reveal a considerable suppression of the creep rate above the second peak supporting the idea about the formation of a new dislocation glass in the entangled solid state.

\section{ACKNOWLEDGEMENTS}

We would like to thank G. Blatter, V. Geshkenbein, M. Konczykowski, and E. Zeldov for numerous fruitful discusions and W.K. Kwok for critical reading of the manuscript. This work was supported from Argonne National Laboratory through the U.S. Department of Energy, BES-Material Sciences, under contract No. W-31109-ENG-38 and by the NSF-Office of Science and Technology Centers under contract No. DMR91-20000 Sci- ence and Technology Center for Superconductivity.

${ }^{1}$ A. I. Larkin, Sov. Phys. JETP 31, 784 (1970).

${ }^{2}$ A. I. Larkin and Y. N. Ovchinnikov, J. Low Temp. Phys. 34, 409 (1979).

${ }^{3}$ M. P. A. Fisher, Phys. Rev. Lett., 62, 1415 (1989).

${ }^{4}$ D. S. Fisher, M. P. A. Fisher, and D. A. Huse, Phys. Rev. B, 43, 130 (1991).

${ }^{5}$ G. Blatter et al., Rev. Mod. Phys. 66, 1125 (1995).

${ }^{6}$ M. V. Feigel'man, V. B. Geshkenbein, A. I. Larkin, and V. M. Vinokur, Phys. Rev. Lett. 63, (1989).

7 T. Nattermann, Phys. Rev. Lett. 64, 2454 (1990).

${ }^{8}$ T. Giamarchi and P. Le Doussal, Phys. Rev. Lett. 72, 1530 (1994); Phys. Rev. B 52, 1242 (1995).

9 J. P. Bouchaud, M. Mezard, and J. S. Yedidia, Phys. Rev. B 46, 14686 (1992).

10 S. Korshunov, Phys. Rev. B 48, 3969 (1993).

${ }^{11}$ B. Jankovici, Phys. Rev. Lett. 19, 20 (1967); N. D. Mermin, Phys. Rev. 176, 250 (1968).

12 S. Ryu, A. Kapitulnik, and S. Doniach, Phys. Rev. Lett. 77, 2300 (1996).

13 M. Gingras and D. A. Huse, Phys. Rev. B 53, 15193 (1996).

${ }^{14}$ R. N. Kleiman Phys. Rev. Lett. 62, 2331 (1989).

${ }^{15}$ R. Cubitt et al., Nature 365, 407 (1993).

${ }^{16}$ K. Harada et al. Phys. Rev. Lett. 71, 3371 (1993).

17 T. T. M. Palstra et al., Phys. Rev. Lett. 61, 1662 (1988); N. Kobayashi et al., Physica C159,295 (1989); R. Busch et al., Phys. Rev. Lett. 69, 522 (1992); J. H. Cho et al., Phys. Rev. B, 50,6493 (1994)).

18 J. Kierfeld, T. Nattermann and T. Hwa, Phys. Rev. B 55, 626 (1997).

${ }^{19}$ V. N. Kopylov, I. F. Shegolev, and T. G. Togonidze, Physica C 162-164, 1143(1989), V. N. Kopylov, A. E. Koshelev, I. F. Shegolev, and T. G. Togonidze, Physica C 170, 291 (1990).

${ }^{20}$ M. Daeumling, J. M. Seuntjens, and D. C. Larbalestier, Nature 346, 332 (1990).

${ }^{21}$ N. Chikumoto et al, Physica C 185-189, 2201 (1991).

22 N. Chikumoto et al, Phys. Rev. Lett. 69, 1260 (1992).

${ }^{23}$ M. A. Angadi et al, Physica C 185-189, 2159 (1991).

${ }^{24}$ K. Kadowaki and T. Mochiku, Physica C 195, 127 (1992).

25 T. Tamegai et al Physica C 213, 33 (1993)

${ }^{26}$ Ming Xu et al.,Phys. Rev. B, 48,10630 (1993); Ming Xu, K. Zhang, and B. Dabrowski, in Studies of High Temperature Superconductors, Vol. 14, edited by A. V. Narlikar, Nova Science Publisher, NY, (1994).

${ }^{27}$ V. Hardy et al. Physica C 232,347 (1994)

${ }^{28}$ E. Zeldov et al., Europhys. Lett. 30, 367 (1995).

${ }^{29}$ B. Khaykovich et al., Phys. Rev. Lett. 76, 2555 (1996).

${ }^{30}$ A. I. Larkin and V. M. Vinokur, Phys. Rev. Lett. 75, 4666 (1995).

${ }^{31}$ K. Kishio et al., in Proc. 7th Interntnl. Workshop on Critical Currents in Superconductors, Ed. H. W. Weber, World Scientific, Singapore (1994), p.339. 
32 T. Tamegai et al., in Proceedings of the 9th International Symposium on Superconductivity (ISS'96), October 21-24, 1996, Ed. S. Nakajima and M. Murakami, Springer-Verlag, Tokyo (1997), p. 621

${ }^{33}$ B. Khaykovich et al., Phys. Rev. B 56, R517 (1997).

${ }^{34}$ S. L. Lee et al., Phys. Rev. Lett. 71, 3862 (1993).

${ }^{35}$ C. M. Aegerter et al., Phys. Rev. B 54, R15 661 (1996).

${ }^{36}$ D. Ertaş and D. R. Nelson, Physica C 272, 79 (1996).

${ }^{37}$ T. Giamarchi, and P. Le Doussal, Phys. Rev. B 55, 6577 (1997), preprint cond-mat/9609112

38 J. Kierfeld, preprint cond-mat/9609045

${ }^{39}$ V. M. Vinokur et al., Preprint, 1997

${ }^{40}$ A. E. Koshelev, L. I. Glazman, and A. I. Larkin, Phys. Rev. B 53, 2786 (1996).

${ }^{41}$ E. H. Brandt and A. Sudbø, Physica C 180, 426 (1991).

${ }^{42}$ A. E. Koshelev and P. H. Kes, Phys. Rev. B 48, 6539 (1993).

43 J. M. Kim and J. M. Kosterlitz, Phys. Rev. Lett. 62, 2289 (1989); B. M. Forest and L. -H. Tang, Phys. Rev. Lett. 62, 2289 (1989); J. M. Kim, A. J. Bray, and M. A. Moore Phys. Rev. A44, 2345 (1991); L. -H. Tang, B. M. Forest and D. E. Wolf, Phys. Rev. A45, 7162 (1992).

44 T. Halpin-Healy, Phys. Rev. Lett. 62, 442 (1989).

45 T. Halpin-Healy and Y. C. Zhang, Phys. Rep. 254, 215 (1995).

${ }^{46}$ L. I. Glazman and A. E. Koshelev, Phys. Rev. B43,2835 (1991).

${ }^{47}$ G. Blatter et al., Phys. Rev. B54, 72(1996).

${ }^{48}$ Y. Abulafia, A. Shaulov, Y. Wolfus, L. Burlachkov, Y. Yeshurun, D. Majer, E. Zeldov, and V. M. Vinokur, Phys. Rev. Lett. 75, 2404 (1995).

${ }^{49}$ M. Konczykowski et al., unpublished

\section{FIGURES}

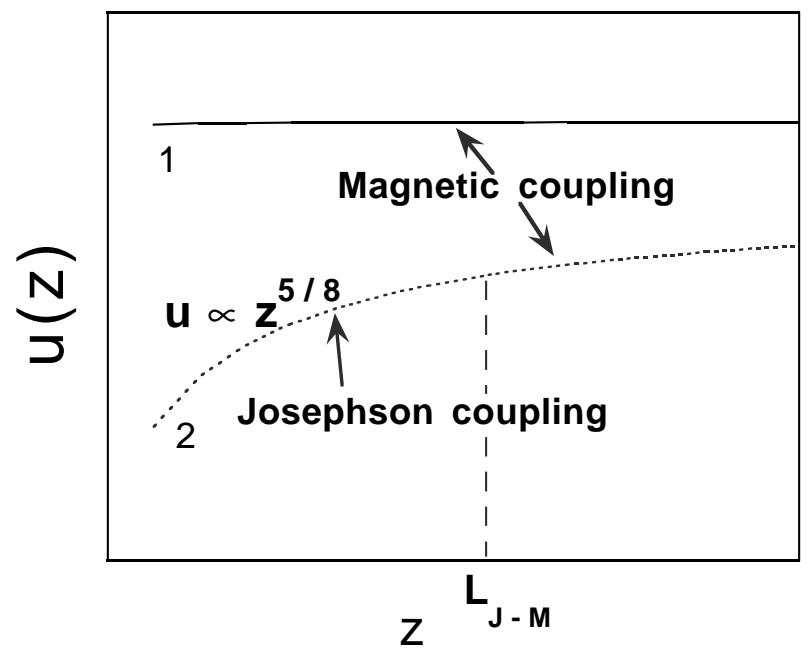

FIG. 1. Regimes of the vortex line wandering in layered superconductors. If only magnetic interlayer coupling is present, then displacements don't grow with distance (curve 1). For moderate Josephson coupling displacements grow as $z^{5 / 8}$ at small distances and saturate at length $L_{\mathrm{J}-\mathrm{M}}$ (curve 2)

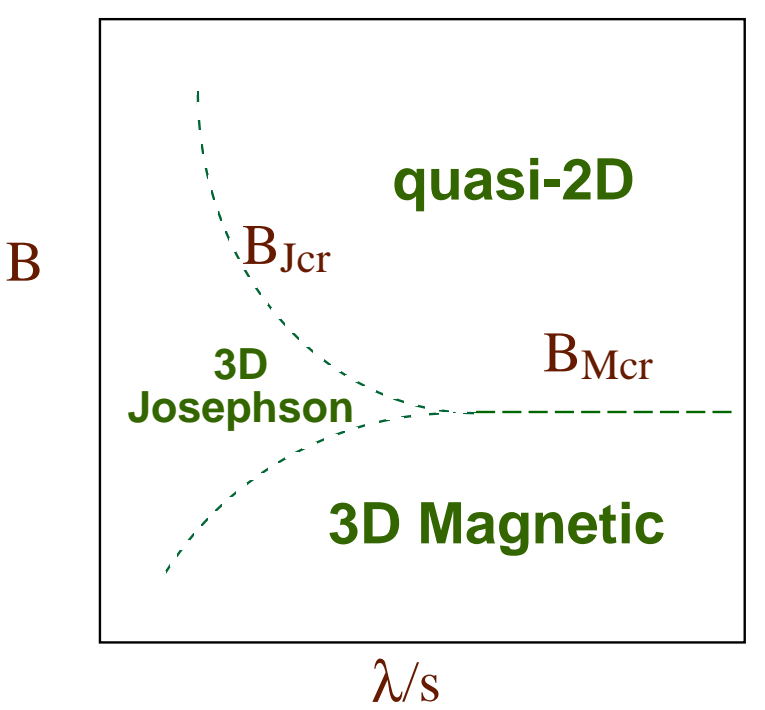

FIG. 2. Different regimes of lattice behavior in the anisotropy-field plane

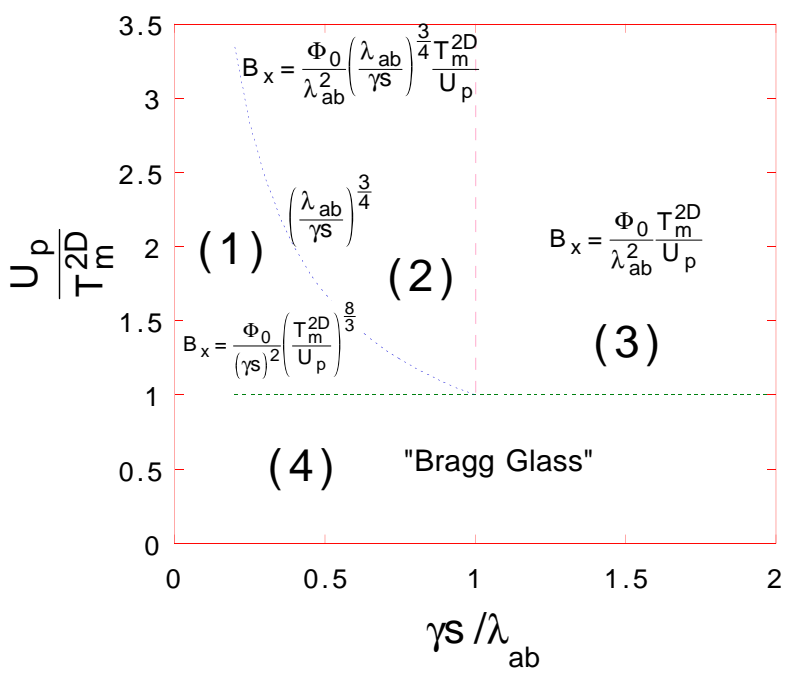

FIG. 3. Different regimes of disorder driven transition in the pinning strength-anisotropy plane: (1) The region of dominating Josephson coupling; (2) The intermediate region (Josephson coupling dominates at small scales, magnetic coupling dominates at the "interaction" length scale); (3) The region of dominating magnetic coupling (4) The region of weak pinning within which the ordered lattice state remains stable up to the upper critical field. Estimates for the transition fields $B_{x}$ are shown for regions (1), (2), and (3) 